\section{LA TERRA RICHIEDE LA NOSTRA CURA}

La lettera di Federico Marolla, che pubblichiamo in questo numero (pag. 488), è a un tempo un atto di accusa e un forte richiamo a tutti noi.

I dati riportati fanno venire i brividi pensando al futuro del nostro pianeta e dei bambini di cui ci occupiamo giornalmente. Solo la frase "alcuni cambiamenti che sono già in atto - come il continuo aumento del livello del mare - sono irreversibili" ci fa pensare alle conseguenze del riscaldamento globale: per le centinaia di milioni di bambini che vivono nelle metropoli situate a pochi metri sul livello del mare; per quelli esposti a eventi metereologici estremi; per altri ancora esposti a migrazioni forzate per sfuggire a siccità, inondazioni, conflitti a loro volta scatenati da competizione per l'acqua o fonti di energia. Senza contare gli aspetti non meno drammatici ma più silenti nella loro evoluzione e meno immediatamente evidenti nelle loro conseguenze, come la perdita di biodiversità. Per chi volesse approfondire, raccomandiamo la lettura nel bellissimo e molto documentato report dell'UNICEF riguardo le conseguenze sui bambini del riscaldamento globale' (dovremmo smettere di chiamarlo cambiamento climatico, denominazione alquanto equivoca imposta in campo internazionale dall'allora - presidenza Bush - vicepresidente USA Dick Cheney, con forti interessi nel settore petrolifero e "negazionista ambientale") - l'appello delle maggiori riviste mediche riportato su Lancet il 6 settembre $2021^{2}$.

Ė giunto dunque il momento in cui tutti noi, ognuno nel suo ruolo, dobbiamo impegnarci per promuovere un cambiamento negli stili di vita, dei singoli e delle famiglie. Questo aspetto non è meno importante delle grandi decisioni prese a livello internazionale, perché senza miliardi di piccoli gesti quotidiani difficilmente quelle grandi azioni saranno comprese e quindi sostenute e sostenibili.

Dobbiamo farlo come Società professionali e scientifiche, mettendo questo tema al centro delle proprie iniziative; dobbiamo farlo come singoli pediatri, rimodulando i nostri stessi ambulatori in alcuni aspetti anche minori, nei messaggi, nei nostri stessi stili di vita e naturalmente sollecitando e accompagnando le famiglie dei nostri assistiti verso una maggiore consapevolezza e verso azioni conseguenti; dobbiamo farlo come semplici cittadini, contribuendo alle politiche dei nostri enti locali.

$\mathrm{C} i$ sono già documenti e iniziative importanti in tal senso. Ricordiamo il documento di consenso "Inquinamento atmosferico e salute" nato nell'ambito del progetto "I primi 1000 giorni", sottoscritto da tutte le principali Associazioni pediatriche italiane (ACP, FIMP, SIMP, SIN, SIP) e presentato ufficialmente a Roma il 6 ottobre 2021, che in 8 punti declina degli impegni precisi delle Società scientifiche su questi temi, tra cui informare pazienti e famiglie e adottare per prime dei comportamenti più sostenibili ${ }^{3}$.

E... c'è una novità: tra i materiali a disposizione dei pediatri per aiutare se stessi, i colleghi e le famiglie dei propri pazienti, oltre al libro-documento del gruppo Pediatri per un Mondo Possibile ${ }^{4}$, c'è ora il libretto, rivolto a bambini, genitori e insegnanti affinché si prendano cura del problema

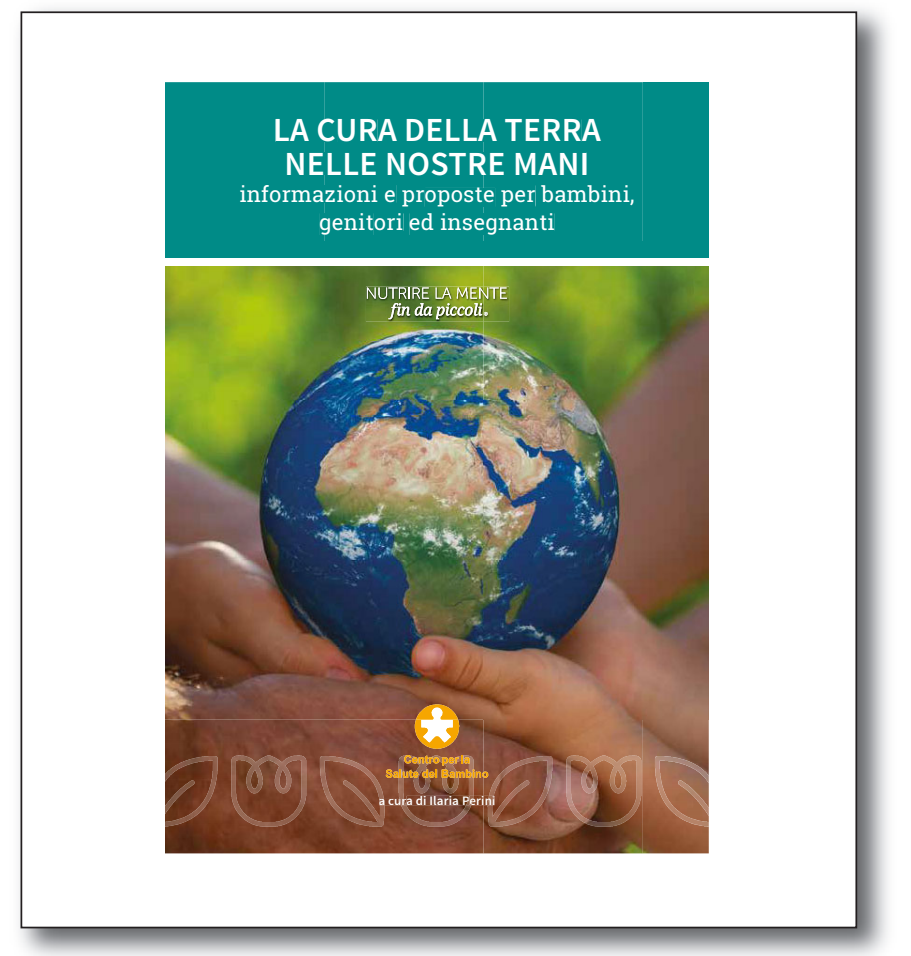

a partire dalle mura domestiche. Il libretto è prodotto dal Centro per la Salute del Bambino (CSB) in collaborazione con il gruppo Pediatri per un Mondo Possibile (PUMP) delI'ACP e l'Osservatorio Geofisico Sperimentale di Trieste, ed è disponibile su ordinazione presso il CSB (www.csbonlus.org). "La cura della Terra nelle nostre mani: informazioni e proposte per bambini, genitori e insegnanti" è il titolo dell'opuscolo che contiene delle schede pratiche ricche di suggerimenti per modificare i propri stili di vita in modo più ecologico e sostenibile (Figura): energia, mobilità, nutrizione, rifiuti, abbigliamento, cosmesi, autoproduzione, educazione in natura, piccole grandi azioni sono i temi del libro, arricchito da schede pratiche utilizzabili sia nelle scuole che nelle famiglie.

Ė tempo di passare tutti all'azione concreta. La Terra richiede la nostra cura.

\section{Bibliografia}

1. UNICEF. Unless we act now: the impact of climate change on children. November 2015.

2. Atwoli L, H Baqui AH, Benfield T, et al. Call for emergency action to limit global temperature increases, restore biodiversity, and protect health. Lancet 2021;398(10304):939-41. doi: 10.1016/ S0140-6736121)01915-2.

3. I primi 1000 giorni. https://millegiorni.info.

4. Toffol G, Todesco L, Reali L. Inquinamento e salute dei bambini. Cosa c'è da sapere, cosa c'è da fare. Seconda edizione. Roma: Il Pensiero Scientifico editore, 2017.

Giacomo Toffol Pediatra di famiglia, Treviso Giorgio Tamburlini Centro per la Salute del Bambino onlus, Trieste 